\title{
Hard Ware Design of Transformer Terminal Unit (TTU) Based on Substation Automation
}

\author{
Ibrahim Mahammed Kilishi ${ }^{1}$, Kassim Bello Muhammad ${ }^{2}$, Abdulaziz Bello ${ }^{3}$ \\ Ibrahim Hussain Alhasan ${ }^{4}$ \\ Department ofElectrical / Electronic, College Liaoning University of Technology, Jinzhou 121001, China ${ }^{1}$ \\ Department of Technical Education (Electrical), School of Technology Kano, Kano State Polytechnic, Nigeria ${ }^{2}$ \\ Department of Electrical / Electronic, College Liaoning University of Technology, Jinzhou 121001, China ${ }^{3}$ \\ Department ofElectrical / Electronic, College Liaoning University of Technology, Jinzhou 121001, China ${ }^{4}$
}

\begin{abstract}
The distribution transformer monitoring system is composed of Transformer Terminal Unit (TTU), GPRS wireless communication network and master station. TTU is installed in the transformer, which mainly accomplishes data collection, analysis and record, pops up alarm information in time when the transformer has abnormal events, receives the command from the master station and implements it. GPRS wireless communication network is the bridge of data transmission between the master station and transformers. Master station receives the real-time data of transformers through GPRS wireless communication network, implements further data processing and saving, consequently issues control command. Therefore the system can achieve a comprehensive monitoring and control for distribution transformers. At the same time, it can provide users with a visual interface and let dispatches obtain monitoring performance of far transformers in time for never leaving home. Choosing an appropriate device should also be considered when designing and low power consumption components. From replacement and easy maintenance considerations and reliability, the controller part is independent chassis equipment. We focus on the selection of hard ware component and corresponding hardware design of TTU, designing a high reliability and multifunction TTU which meet the application requirements is an urgent need for substation automation. This paper presents a new hardware design for TTU; the design is composed of seven main parts with its own components which include: conditioning circuit of A-Phase voltage and conditioning circuit of A-Phase current, Circuit diagram of switch input. power supply regulator, Multiplexer switch (AD7506), Clock circuit design output switch control circuit and Communication interface circuit.
\end{abstract}

Keywords: CPU (STC12C5620AD), hard ware, Substation automation, TTU

\section{Introduction}

TTU transformer terminal unit is an effective device to monitor the running state of transformers in the substation automation system. TTU is an important component of substation automation system. It is the remote terminal which is installed in the bottom of the system and used for monitoring the real-time operation parameters of the distribution transformers. under the market demand of distribution transformers, it is therefore desirable to design a monitoring device which can collect the data precisely; meanwhile it can communicate with the remote terminals and monitoring centre reliably, TTU is designed for the protection, control, measurement and supervision of two-winding power transformers and power generator-transformer blocks in distribution networks. The new TTU can measure the electric parameters of the distribution transformer precisely. Taking advantage of the powerful arm processor, it can analyses harmonic of the power line effectively. Due to the always-on-line feature of GPRS, TTU can achieve reliable communication with the remote terminal and the master station. Compared with other similar units, the new unit performs in terms of real-time, precision and reliability, which can fully meet with the high-speed development of substation automation system.

The main function of TTU

(1) Measurement capabilities: TTU can measure three-phase voltage, current, harmonic current, active power, reactive power, power factor, etc.

(2) Recording capabilities: TTU can record all measured values of the parameters mentioned above which includes minimum value and maximum value.

(3) Statistical capabilities: TTU can make a statistical investigation of blackout time in one year, voltage quality rate, switching times of capacitor, etc.

(4) Analysis functions: TTU can complete online and offline analysis of current curves, voltage quality, harmonic distortion rate, etc. and give corresponding governance methods. 
(5) Protection functions: TTU can provide an alarm signal in real time. It has perfect communication function and operation record function, and can record all the operation events for analysis.

(6) TTU can control the switching of capacitors accurately; keep the three-phase low-voltage balance of transformer.

\section{Hardware Design of TTU}

TTU has many integrated functions of remote control measurement and remote communication and also acquire high requirements for accuracy measurement and reliability. In this design the method of data collection is by sampling AC signal from Potential Transformer and Current Transformer (PT \& CT). TTU design is as follows: Voltage and current signal flowed into the corresponding sensors, then the filter circuit, sample hold circuit, multiplex switch, A/D conversion, and finally entered into the CPU. The communication module mostly adopts RS-485 Bus or other wired communication mode. In general the TTU hard ware design requires some components in integration for proper and accurate system positive workout. The following components were taken into consideration for analog data sampling and processing; AC quantity data sampling, digital data acquisition circuit, digital output or (switch output) circuit, communication interface, CPU, RAM and other various intelligent electronic devices. The components of design are as follows:-

1. $\quad$ CPU: using (STC12C5620AD)

2. Design of an Analog detection circuit using (TL085).

3. Selection of a Sample and Hold circuit (LF398)

4. Appropriate selection of a DC-DC converters $(+5 \mathrm{~V}$ and $\pm 12 \mathrm{~V})$

5. $\quad$ Communication interface module (MAX485)

6. $\quad$ Optical Isolation circuit (TLP521-1)

7. $\quad$ External Flash memory RAM (AT24C64)

8. $\quad$ Multiplexer switch (AD7506)

9. $\quad$ Sensor.(PT 100)

All of the above component were perfectly selected, Also with the application of the Altium designer software the schematic diagram of all parts of the system was designed respectively, which includes:-

\section{[1] Current and Voltage Signal Conditioning Circuit}

In the three-phase current signal collections, phase current IA is changed into suitable voltage signal for measuring range of chip by $10 \mathrm{~A} / 10 \mathrm{~mA}$ current transformer and resistance. $1.2 \mathrm{k} \Omega$ resistance and $0.01 \mathrm{uF}$ capacitance constitute the anti-aliasing filter and the voltage signal after filtering flows into the current input channel of ATT7022B. The principle of three phase voltage signal collections is the same as three phase current collection circuits. Because the voltage transformer adopts $2 \mathrm{~mA} / 2 \mathrm{~mA}$ current-mode voltage transformer, voltage UA needs to be changed into $2 \mathrm{~mA}$ current signal by the resistor R1 firstly. The current and voltage conditioning circuits are the same except voltage condition circuit used voltage transformer while current conditioning circuit used current transformer. Figure: 1showsconditioning circuit of A-Phase voltage.

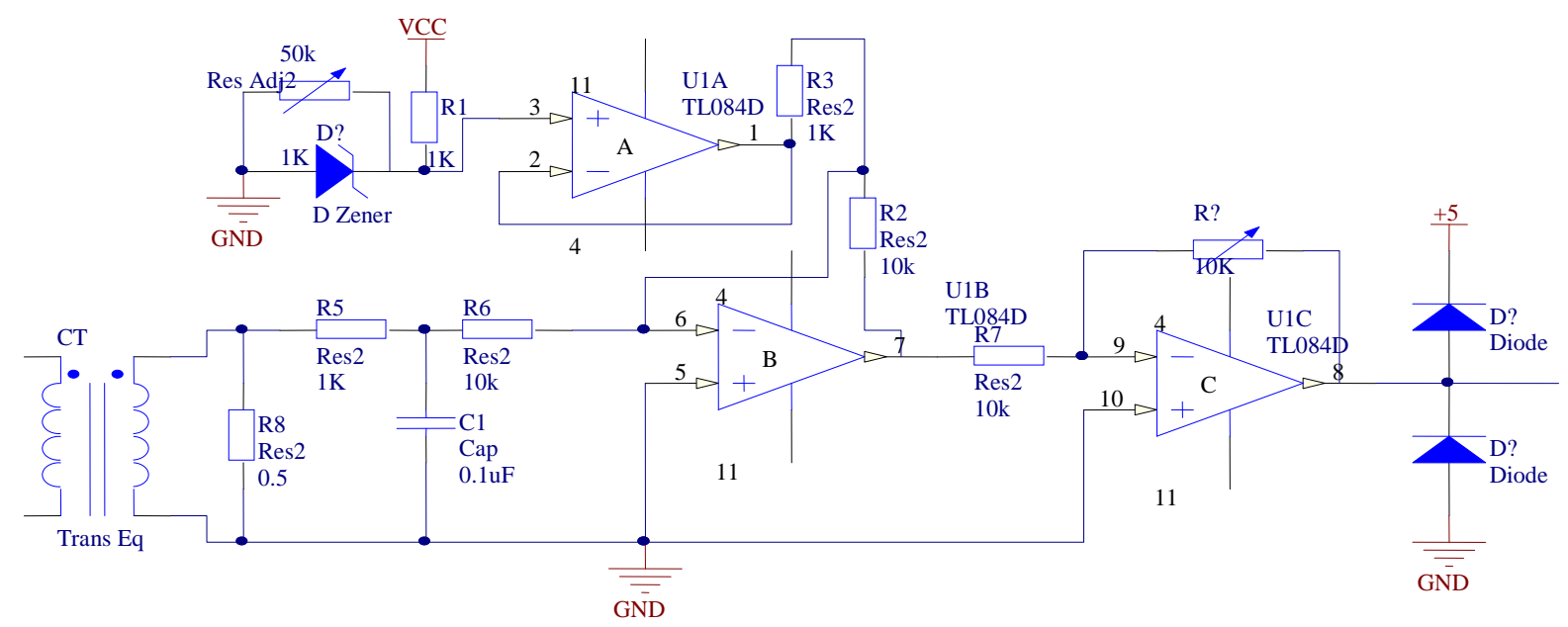

Figure: 1 conditioning circuit of A-Phase voltage

[2] Design of a TTU input condition monitoring circuit 
Switch input signal of TTU mainly includes remote signal and remote pulse signal. Remote signal indicates the position status of the circuit breaker of the distribution transformer and the action status of protection and automatic devices. Remote pulse signal is to read the pulse signal of multi-function electric meter. Switch input signal must be converted to $3.3 \mathrm{~V}$ logic signal by the isolation of the auto coupler first and then enters into I/O port of (LF398). The circuit of the switch input module is shown in Figure: 2

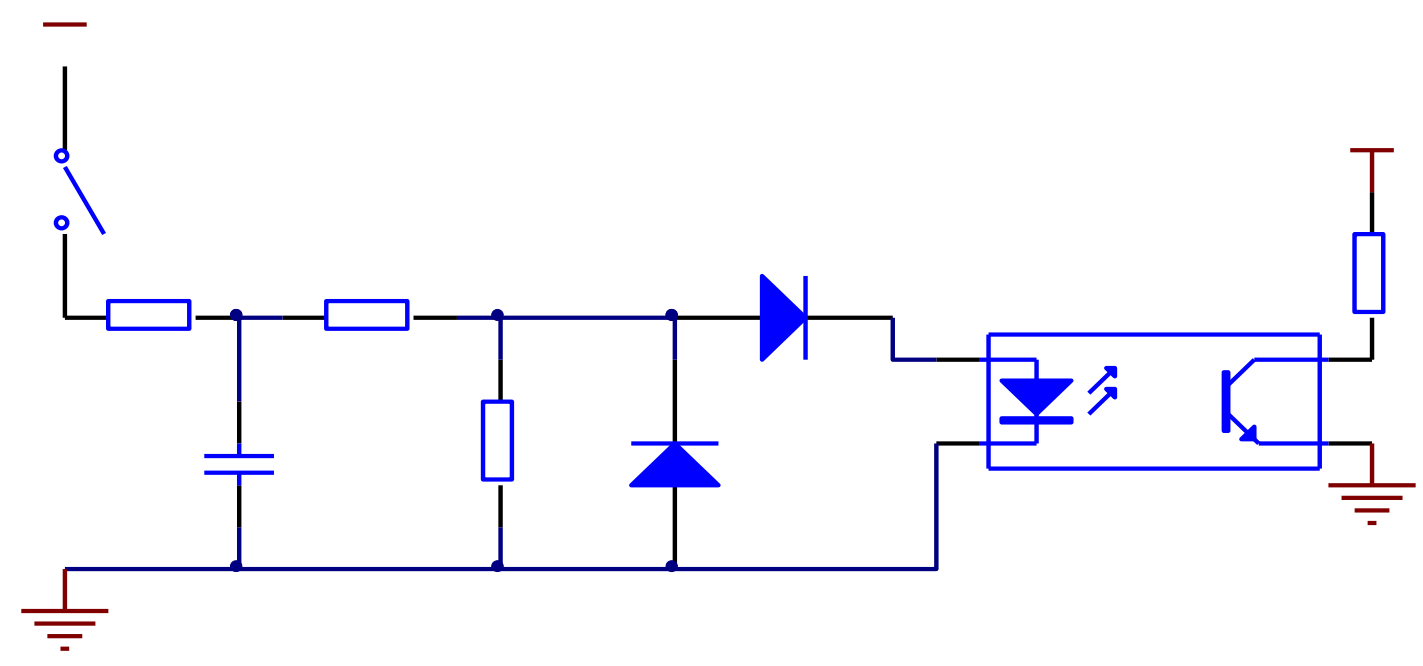

Figure: 2 Circuit diagram of switch input.

[3] Design of TTU power supply regulator

A power supply is a device that supplies electric power to an electrical load. The term is most commonly applied to electric power converters that convert one form of electrical energy to another. A power supply regulator is one that controls the output voltage or current to a specific value; the controlled value is held nearly constant despite variations in either load current or the voltage supplied by the power supply's energy source In this design there were two different DC regulated power needed by different electronic equipment in accordance with their power consumption specification provided from their data sheet namely; $5 \mathrm{~V}$, and $\pm 12 \mathrm{~V}$.

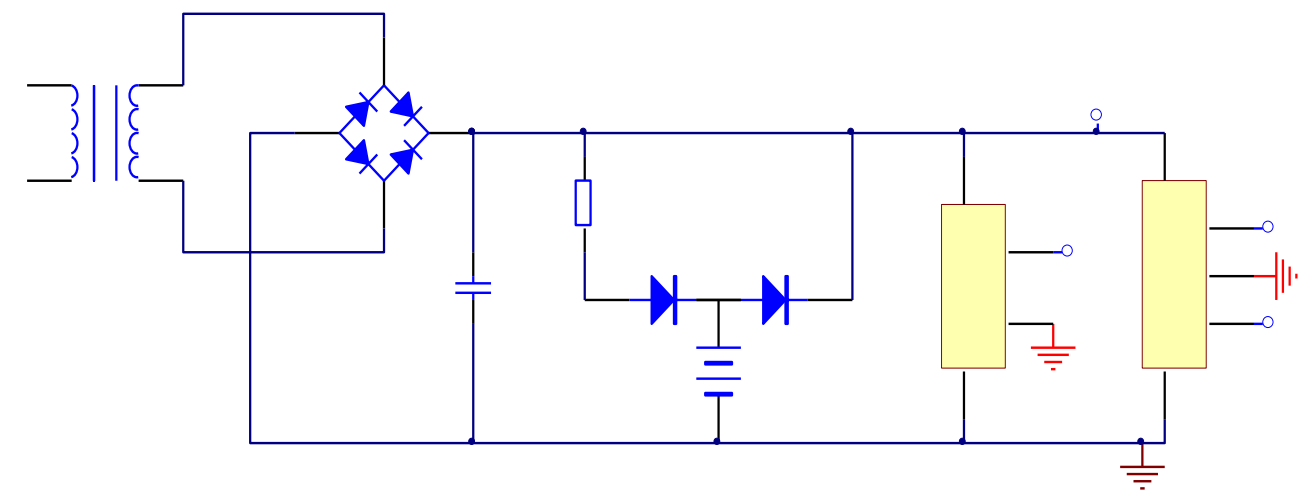

Figure: 3 Design of TTU power supply regulator

[4] Multiplexer switch (AD7506)

The AD7506 is a monolithic CMOS 16-channel analog multiplexer packaged in a 28-terminal surface mount package. It switches a common output to one of 16 inputs, depending on the state of four address lines and an "enable" The AD7506 is a monolithic CMOS 16-channel analog multiplexer packaged in a 28-terminal surface mount package. It switches a common output to one of 16 inputs, depending on the state of four address lines and an "enable". The AD7507 is identical to the AD7506 except it has two outputs switches to two of 16 inputs depending on three binary address states and an "enable". A rudimentary way of calculating how long a switch or multiplexer takes to settle can be estimated by calculating the RC for the device, that is, $\mathrm{RON} \times \mathrm{CD}$, and multiplying by the number of time constants for required system accuracy. This is added to the switch timing, TON, TOFF, or transition, for the switch or multiplexer. 


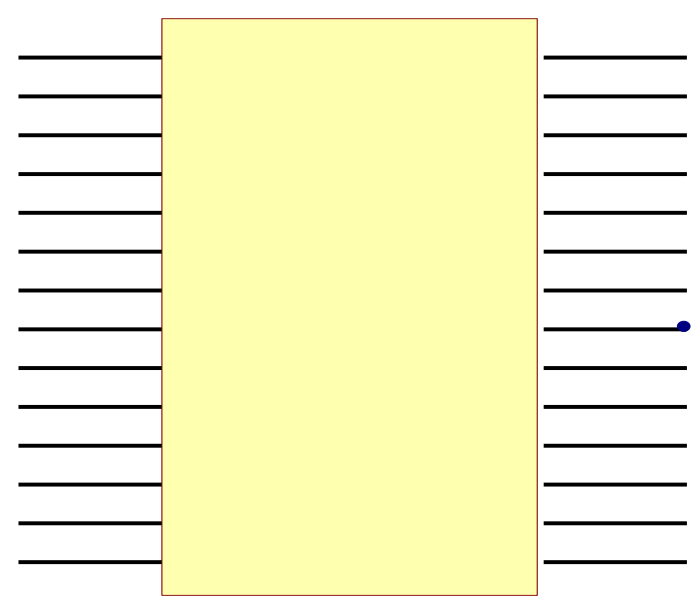

[5] Design of TTU clock circuit

Fig4 Multiplexer switch (AD7506)

The heart of the STC12C5A60S2 is the circuitry that generates the clock pulses by which all the internal operations are synchronized. Pins XTAL1 and XTAL2 are provided for connecting a resonant network to form an oscillator. Typically a quartz crystal and capacitors were employed in this clock design. The STC12C5A60S2 MCU requires a basic crystal frequency for its internal frequency generation and control.

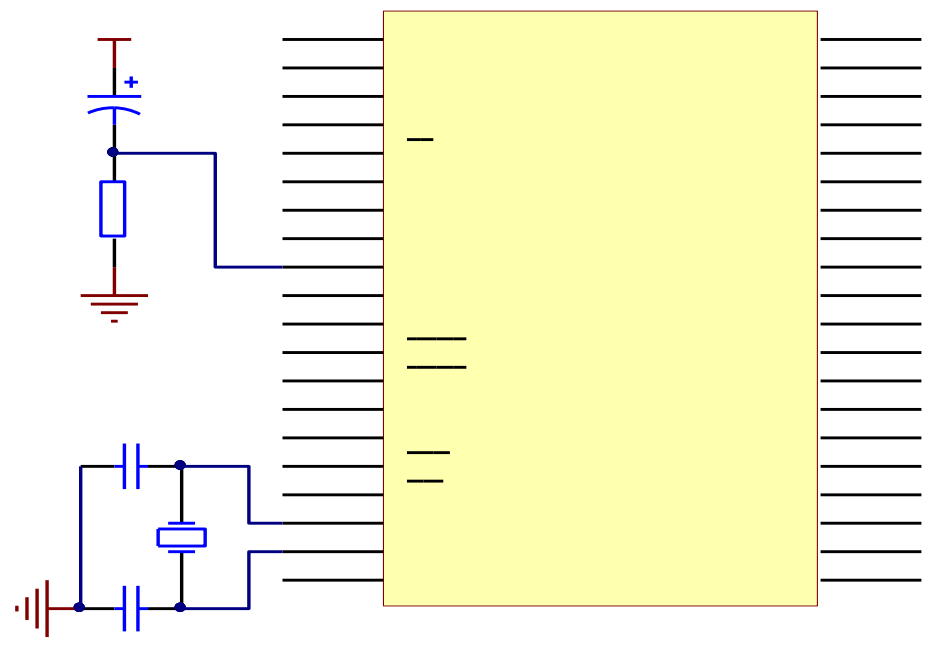

Figure: 5 Clock Circuit Design

[6] Design of TTU output switch control circuit

TTU output switch control circuit is also known as remote output switch circuit, is the essential part of the TTU interface for execution and implementation of feeder safety, security and control. The design of the digital output from the software and hardware should consider logic gates to export optimal control in order to ensure reliability of operation. Remote output circuit should provide an appropriate feedback, due to mechanical actuators delay to respond and execute control action instantly, the functions are protection, tripping operation the local and central signals and communication structure is to isolated the Internal and external by the photoelectric coupling, use XOR logic control state, To prevent the disoperation at the time of starting or shutdown. 


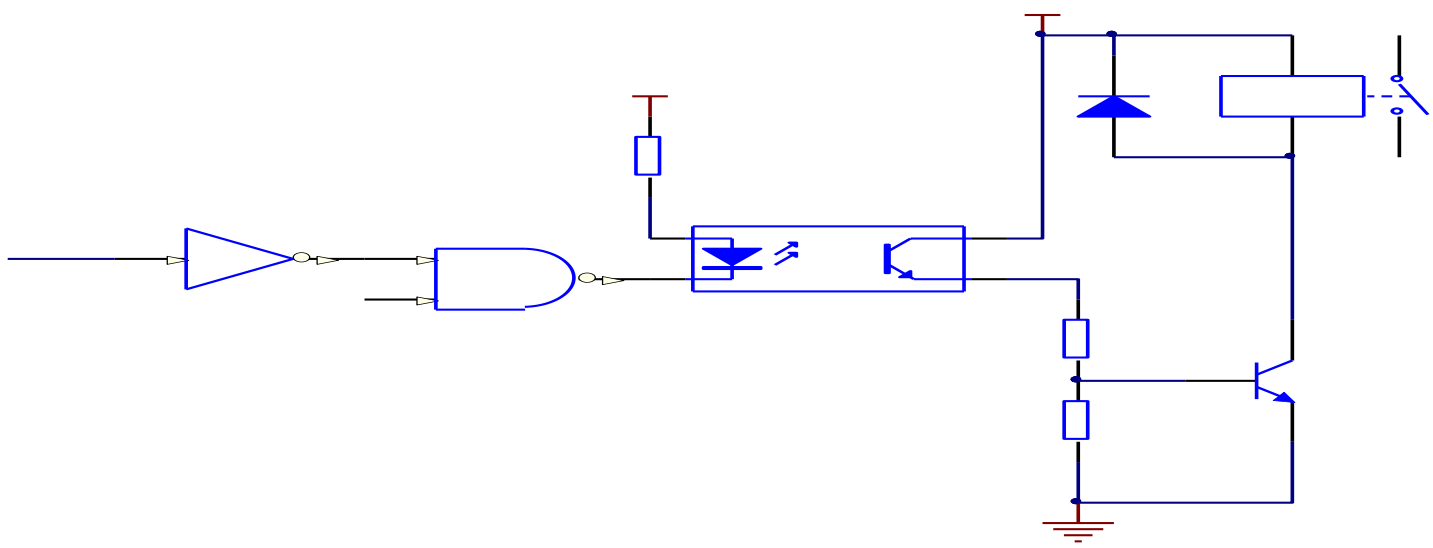

Figure: 6 Design of TTU output switch control circuit

[7] Design of a communication interface

A communication channel should be selected to interface between the remote control devices and the CPU for real-time monitoring and control of substation. In this paper a MAX485 is selected to serve as the communication interface between the CPU and remote device control between the CPU and remote control devices. The MAX485 is low-power transceivers for RS-485 and RS-422 communication. The IC contains one driver and one receiver. The driver slew rates of the MAX485 is not limited, allowing them to transmit up to $2.5 \mathrm{Mbps}$. These transceivers draw between $120 \mu \mathrm{A}$ and $500 \mu \mathrm{A}$ of supply current when unloaded or fully loaded with disabled drivers. All parts operate from a single 5V supply.

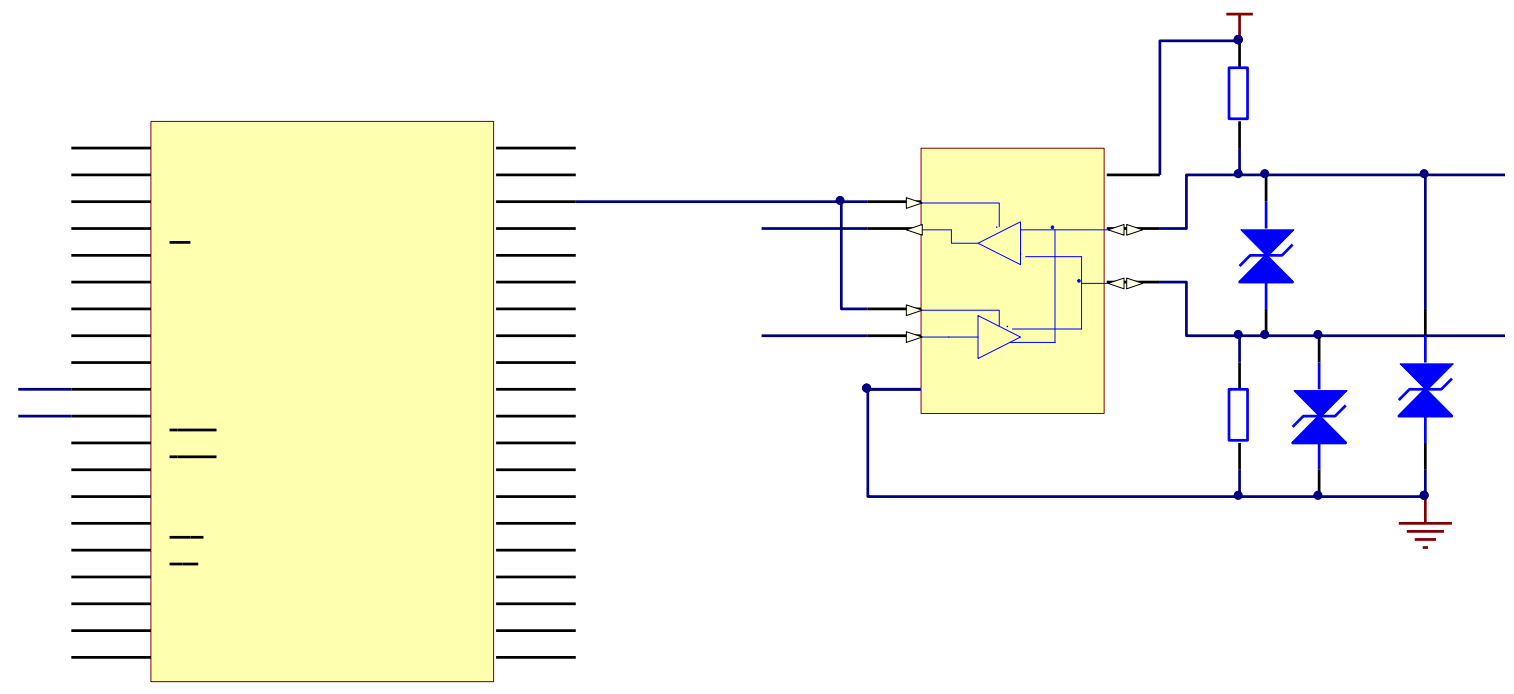

Fig7 Communication interface circuit

\section{Conclusion}

TTU is an important component of distribution automation system. It is the remote terminal which is installed in the bottom of the system and used for monitoring the real-time operation parameters of the distribution transformers. Under the market demand of distribution transformers, it is therefore desirable to design a monitoring device which can collect the data precisely; meanwhile it can communicate with the remote terminals and monitoring centre reliably. TTU has integrated many functions of remote control measurement and remote communication and also acquire high requirements for accuracy measurement and reliability, the hardware designed composed of seven main parts with its own components which include: conditioning circuit of A-Phase voltage and conditioning circuit of A-Phase current, Circuit diagram of switch input. power supply regulator, Multiplexer switch (AD7506), Clock circuit design output switch control circuit and Communication interface circuit. The entire component was perfectly selected, also with the application of the Altium designer software the schematic diagram of all parts of the system was designed respectively. The hardware designed of TTU in this paper can also be further improved and the below development directions can be taken; the design of the PT100 Sensor circuit design of the Alarm Module etc. 


\section{Acknowledgment}

We would like to acknowledge the assistance given by all the members of the Department of Electrical/Electronic, Liaoning University of Technology China, for going through the paper. Our special thanks go to Professor Ren Guo Chen for the support and assistance given to this research.

\section{References}

[1]. Na Wu, YinjingGuo, Study New Smart Transformer Terminal Unit on Based on ARM and GPRS Network, DECEMBER 2013

[2]. MaXiaoLu, the Study of Transformer Terminal Unit Based on MSP430, 2005

[3]. LI Guang-hu, Study of DSP based transformer terminal unit (TTU), 2004

[4]. B.L. Therajaet. all "A Textbook of Electrical Technology Vol.III, Transmission, Distribution and Utilization," S. Chand \& Co. Ltd., 2002.

[5]. David Mark Edward Ingram, Assessment of Precision Timing and Real-Time Data Networks for Digital Substation Automation, February 2013

[6]. Bruce Carter and Thomas R. Brown, Handbook of Operational Amplifier Applications, sboa092a-October 2001

[7]. Dr. C.K Chan, Design and development STC MCU: ISP Programming

[8]. National semi conductor, LF198/LF298/LF398, LF198A/LF398AMonolithic Sample-and-Hold Circuits, July 2000

[9]. http://www.analog.com/static/imported-files/data_sheets/AD7506.pdf8 and 16 channel analog multiplexers, AD7506/AD7507

[10]. http://www.lan23.ru/wifi/vswr/TL084.pdf 